\title{
(2) OPEN ACCESS \\ Surveillance of people with previously successfully treated diabetic macular oedema and proliferative diabetic retinopathy by trained ophthalmic graders: cost analysis from the EMERALD study
}

\author{
Mandy Maredza, ${ }^{1}$ Hema Mistry, ${ }^{1}$ Noemi Lois 0 , ${ }^{2}$ Steve Aldington, ${ }^{3}$ Norman Waugh, ${ }^{1}$ \\ on behalf of the EMERALD Study Group
}

\begin{abstract}
- Additional online supplemental material is published online only. To view, please visit the journal online (http://dx.doi.org/10.1136/ bjophthalmol-2021-318816).
\end{abstract}

${ }^{1}$ Warwick Medical School, University of Warwick, Coventry, UK

${ }^{2}$ The Wellcome-Wolfson Institute for Experimental Medicine, Queens University Belfast, Belfast, UK

${ }^{3}$ Gloucestershire Hospitals NHS Foundation Trust, Gloucester, UK

\section{Correspondence to}

Dr Mandy Maredza, Warwick Medical School, University of Warwick, Coventry CV4 7AL, West Midlands, UK;

M.Maredza@warwick.ac.uk

Received 8 January 2021

Accepted 5 May 2021
Check for updates

(C) Author(s) (or their employer(s)) 2021. Re-use permitted under CC BY. Published by BMJ.

To cite: Maredza M, Mistry H, Lois N, et al. Br J Ophthalmol Epub ahead of print: [please include Day Month Year]. doi:10.1136/ bjophthalmol-2021-318816

\begin{abstract}
Background/aims Surveillance of people with previously successfully treated diabetic macular oedema (DMO) and proliferative diabetic retinopathy (PDR) adds pressure on ophthalmology services. This study evaluated a new surveillance pathway entailing multimodal imaging reviewed by trained ophthalmic graders and compared it with the current standard care (face-to-face evaluation by an ophthalmologist).

Methods Cost analysis of the new ophthalmic grader pathway, compared with the standard of care, from the perspective of the UK National Health Service, based on evidence from the Effectiveness of Multimodal imaging for the Evaluation of Retinal oedema And new vesseLs in Diabetic retinopathy study. Resource use data were prospectively obtained including times to undertake each procedure. Effectiveness was assessed in terms of sensitivity and specificity of referral decisions in the grader pathway. Costs (SDs) were analysed per 100 patients separately for DMO and PDR at 2018/2019 costs.

Results For DMO, where sensitivity was very high (97\%), the cost difference (savings) for the grader's pathway would be $£ 1390$ per 100 patients. For PDR, the cost would be reduced by $£ 461$ for seven-field Early Treatment for Diabetic Retinopathy Study (ETDRS) images and by $\mathrm{f} 1889$ for ultrawide field images, per 100 patients. Ultrawide images required less time to be obtained and read than seven-field ETDRS. The real savings would be in ophthalmologist time, which could be then redirected to the evaluation of people at high risk of visual loss.

Conclusions Surveillance of people with previously successfully treated DMO and PDR by trained ophthalmic graders can achieve satisfactory results and release ophthalmologist time.

Trial registration numbers NCT03490318, ISRCTN 10856638.
\end{abstract}

\section{INTRODUCTION}

Retinal complications of diabetes, namely diabetic macular oedema (DMO) and proliferative diabetic retinopathy (PDR), can lead to sight loss. In many people, DMO and PDR are treated successfully with laser photocoagulation and/or antivascular endothelial growth factor (anti-VEGF) therapies. However, both can recur following successful treatment, so long-term surveillance is necessary.
The number of people with diabetes is increasing and the numbers attending ophthalmology clinics continue to rise, despite improvements in glycaemic control and screening for DR have reduced the risk of advanced retinopathy. ${ }^{1}$ This makes it difficult for ophthalmology clinics to meet the demand and, as a result, detrimental delays in the evaluation and treatment of patients occur. ${ }^{2}$

National Institute for Health and Care Excellence (NICE) recommends treatment of severe DMO (with central retinal thickness on spectraldomain optical coherence tomography (SD-OCT) of $\geq 400 \mu \mathrm{m}$ ) with anti-VEGFs after which patients should usually be followed monthly during the first year of treatment, and every 1-3 months thereafter to detect recurrence. ${ }^{34}$ In less severe cases, NICE advices macular laser, which is applied in a single session and patients followed subsequently every 3-4 months.

PDR is currently treated with laser panretinal photocoagulation (PRP); treatment with anti-VEGF drugs is being introduced. ${ }^{5}$ After PRP, patients are followed at 6-12 monthly intervals to detect recurrence.

Given the chronic nature of DMO and PDR and the possibility of recurrence, follow-up is lifelong.

Currently in the National Health Service (NHS), ophthalmologists assess patients during follow-up using SD-OCT for DMO and fundus examination by slit-lamp biomicroscopy for PDR. Retinal photographs are not routinely taken to evaluate PDR.

Standard cameras cannot image the retinal periphery and much of the retina is not photographed in the standard seven-field Early Treatment for Diabetic Retinopathy Study (ETDRS) images. Newer ultrawide field (UWF) imaging (Optos, Dunfermline, Scotland, UK) captures nearly the entire retina in a single image; with three images (centre, superior and inferior) the whole retina is covered.

The Effectiveness of Multimodal imaging for the Evaluation of Retinal oedema And new vesseLs in Diabetic retinopathy (EMERALD) study assessed the diagnostic accuracy of a new surveillance pathway (ophthalmic grader pathway) compared with the current standard of care (ophthalmologist face-to-face examination) for people with previously successfully treated DMO and/or PDR. Diagnostic accuracy results of 
EMERALD have been reported. ${ }^{6}$ Herein, we present cost analyses, comparing the ophthalmic grader and standard of care pathways. If the grader pathway is as sensitive at detecting reactivation, graders could take over much of the routine surveillance. Specificity is also important, since the proportion referred by graders to ophthalmologists determines the savings in their time. We also compare costs of UWF fundus images with those of seven-field ETDRS.

\section{PATIENTS AND METHODS Study background}

EMERALD was a diagnostic accuracy study $^{7}$ in adults with type 1 or 2 diabetes and previously successfully treated DMO/ PDR who, at the time of enrolment, had reactivated or inactive disease, recruited from 13 ophthalmology departments in the UK. The primary outcome was sensitivity of the ophthalmic grader pathway to detect presence of DMO and active PDR, using standard care as reference standard, which included slit-lamp examination and review of SD-OCT scans by an ophthalmologist.

The ophthalmic grader pathway entailed review of SD-OCT scans to detect DMO, and seven-field ETDRS and UWF images to detect PDR, by trained, tested and certified ophthalmic graders. The analysis took into consideration ophthalmic graders referring to ophthalmologists all patients diagnosed as having DMO and/or active PDR, or if they were uncertain about the diagnosis, or if images were ungradable, as this is what would occur in clinical practice if the pathway is implemented. As new vessels may be seen on fundus images but missed on fundus examination, the study included an enhanced reference standard for PDR. The latter combined results of face to face examination by the ophthalmologists with the evaluation of seven-field ETDRS and UWF images by an ophthalmologist expert in DR; if any detected active PDR, the grading, based on the enhanced reference standard, was active PDR.

\section{Cost analysis}

Costs of standard care, ophthalmic grader pathway and enhanced reference standard included staff costs (based on time undertaken to perform activities/procedures), equipment costs and costs of training graders. The cost analysis was undertaken from the perspective of the UK NHS. ${ }^{3}$ All costs were at a single time point, expressed in $£$ sterling and valued in 2018-2019 prices.

\section{Measurement and valuation of resource use}

Resource use was captured prospectively on case report forms (CRFs) at each participant's routine standard care visit. CRFs captured the time taken to:

- Undertake best-corrected visual acuity.

- Obtain SD-OCT, seven-field ETDRS and UWF images by ophthalmic photographers/imaging technicians.

- Undertake face-to-face examination by the ophthalmologist, including slit-lamp biomicroscopy, review of SD-OCT scans (to detect DMO) and counselling the patient. However, for the base-case analysis, we used the NHS standard reference cost for an Ophthalmology outpatient visit to cost the standard care pathway. That unit cost captures the reading of SD-OCT by ophthalmologists.

- Grade SD-OCT, seven-field ETDRS and UWF images by ophthalmic graders.

- Grade seven-field and UWF images by the ophthalmologist to set the enhanced reference standard.

Times were converted into staff costs by multiplying the number of hours that staff worked by their hourly salary. We obtained hourly staff costs from the Personal Social Services Research Unit's (PSSRU's) Unit Costs of Health and Social Care compendium $^{8}$ and NHS reference costs. ${ }^{9}$ Graders were costed at band 7 salary scale while photographers/imaging technicians at band 6 level (online supplemental appendix, table A1). All staff costs reflect NHS salary, superannuation, national insurance and overhead costs.

To calculate an annual equivalent cost of equipment, we annuitised capital costs of the item over its useful life span, applying a discount rate of $3.5 \%$ per annum (online supplemental appendix, table A2). ${ }^{10}$ Equipment cost (including annual maintenance costs) was used to derive a per-patient cost of equipment assuming that outpatient throughput for the eye department is 9000 patient visits per year.

Costs of training graders were based on the training delivered in EMERALD to ophthalmic graders and included: (1) costs of preparing training materials; (2) time costs of two trainers delivering a face-to-face meeting over 2 days; (3) salary costs of graders for 2 days; (4) costs of travel to attend the face-to-face meeting; (5) costs of half-day web-based 'further' training for all graders, (6) costs of half-day web-based training for graders who failed the test at first attempt (who had the opportunity to retake it again) and (7) costs of live webinar licence. To follow recommendation that investment costs of education should always be included when evaluating the cost-effectiveness of different approaches to using health service staff ${ }^{11}$ we discounted training costs at 3.5\% p.a., assuming effects of training received will last approximately 10 years. Further details on how graders were trained are reported elsewhere. ${ }^{6}$

\section{Sensitivity analyses}

We compared costs of delivering the pathways based on the main analysis but also based on other scenarios (eg, specifically detecting active disease), as prespecified in the EMERALD Study Statistical Analysis Plan (online supplemental appendix, table A3). In addition, we conducted an exploratory evaluation to assess graders' performance in referring active PDR against an enhanced reference standard consisting of the ophthalmologist face-to-face examination supplemented by reading of retinal photographs. For costing purposes, costs of the enhanced reference standard included reading only one set of images, as this is how it would be done if the pathway were to be implemented.

\section{Analyses of cost data}

We estimated mean costs and SDs of each procedure, including obtaining and reading images (UWF, OCT and seven-field ETDRS). The costs (SDs) are presented per 100 patients. For the standard care pathway costs are simply the reference standard costs for an ophthalmology outpatient visit multiplied by 100 , which include taking and reading SD-OCT images. Costs for the grader pathway options take into account the specificity. Thus, if the specificity of the grader pathway was $50 \%$, half the patients with no active disease would be seen by ophthalmologists. The final costs for the grader pathway would therefore include the costs of photographer taking images + grader reading images for 100 patients + ophthalmologists' time of reviewing patients classified as possibly active DMO/PDR when DMO/PDR is actually inactive, based on specificity.

\section{Missing data}

EMERALD was a cross-sectional study with no follow-up. Images were taken on the same day patients saw the ophthalmologist 
Table 1 Time (in minutes) taken to complete specified activities per patient by ophthalmic photographers/imaging technicians, ophthalmic graders and ophthalmologists

\begin{tabular}{|c|c|c|c|c|c|c|c|}
\hline Time-related activity & $\mathrm{N}$ & Mean & SD & Median & Q1 & Q3 & IQR \\
\hline Standard care** & 380 & 15.3 & 7.6 & 14.0 & 10.0 & 19.0 & 9.0 \\
\hline Ophthalmic photographer/imaging technician obtaining seven-field ETDRS images & 267 & 15.1 & 8.4 & 13.0 & 10.0 & 18.0 & 8.0 \\
\hline Ophthalmic photographer/imaging technician obtaining UWF images & 272 & 10.3 & 6.6 & 9.0 & 5.0 & 14.0 & 9.0 \\
\hline Ophthalmic grader reading seven-field ETDRS images & 267 & 10.6 & 6.5 & 9.0 & 7.0 & 13.0 & 6.0 \\
\hline Ophthalmic grader reading UWF images & 264 & 9.3 & 5.9 & 8.0 & 5.0 & 11.5 & 6.5 \\
\hline Ophthalmologist reading seven-field ETDRS images & 268 & 11.9 & 5.00 & 11.0 & 9.0 & 15.0 & 6 \\
\hline Ophthalmologists reading UWF images & 268 & 10.1 & 4.0 & 9.5 & 7.0 & 12.0 & 5.0 \\
\hline Ophthalmic grader reading SD-OCT images & 248 & 4.2 & 3.3 & 4.0 & 3.0 & 5.0 & 2.0 \\
\hline
\end{tabular}

The standard of care pathway includes all patients $(n=380$ of the full total of 397 in EMERALD; 17 are not included as the times provided for some of the procedures were considered not plausible, being either too high or too low). Note there were 272 patients eligible for grader pathway with DM0 and 281 eligible with PDR.

*Ophthalmologists face-to-face evaluation of patients with access to SD-OCT images.

EMERALD, Effectiveness of Multimodal imaging for the Evaluation of Retinal oedema And new vesseLs in Diabetic retinopathy; ETDRS, Early treatment diabetic retinopathy study;

Q1, Quartile 1; Q3, Quartile 3; SD-OCT, spectral domain optical coherence tomography; UWF, ultra wide field.

and CRFs were completed then. Thus, lost to follow-up was not an issue. In a few individuals recorded values seemed unrealistic, for example, where times to perform a task were close to 0 or more than $60 \mathrm{~min}$ and when images were missing. We excluded these from the analysis.

\section{Longer-term economic modelling}

The study protocol allowed for decision-analytical modelling to estimate the longer-term cost-effectiveness of the ophthalmic grader pathway. Use of a lifelong time horizon may be warranted if observed differences in sensitivity between the new pathway tested and the current standard care were sufficient to affect visual outcomes for either PDR or DMO. We concluded that long-term modelling was not necessary, as sensitivity of the new pathway was similar to that of current standard care for DMO. For PDR, the overall sensitivity was lower but considered acceptable, especially for high-risk PDR. In addition, there are data gaps for several key items, including rate of progression and visual consequences of re-activated PDR in people previously treated with PRP, and the proportion that would be picked up at the next routine visit.

\section{RESULTS}

Table 1 shows the average and median time taken in minutes to complete specified procedures by ophthalmologists, graders and imaging technicians.
Table 2 presents costs of procedures. Costs for obtaining and reading images were lower with UWF compared with seven-field ETDRS. We estimated an annual discounted cost of training graders of $£ 198.70$ per grader, but this cost is trivial once divided by the number of patient images that graders would read in a year.

\section{Costs of surveillance of people with DMO and PDR}

DMO: comparison of ophthalmic grader pathway with current standard care

Table 3 shows costs of follow-up of DMO by ophthalmic graders and ophthalmologists. The cost analysis takes into account referrals by the grader, which would include those related to 'active DMO' (ie, grader believes there is recurrence of DMO), 'unsure' (ie, grader thinks there may be recurrence of DMO but is not fully sure) and 'ungradable' (ie, reader is unable to grade the image). The estimated mean cost of surveillance of people with DMO per 100 patients was $£ 4410.00$ for ophthalmic graders compared with $£ 5800.00$ for standard care. The mean difference (cost savings) per 100 patients would be $£ 1390.00$. The real savings would be in ophthalmologist time, released for other purposes. It should be noted that savings would only apply if neither eye had DMO nor active PDR as otherwise the patient would still require an ophthalmologist evaluation.

All sensitivity analyses (SENA) (online supplemental appendix, tables A4-A7) supported the main finding that surveillance by

Table 2 Costs of procedures

\begin{tabular}{lll}
\hline Item & Cost per patient (SD) & Notes \\
\hline Standard care* (base case) & $£ 58.00$ & $\begin{array}{l}\text { NHS 2019/2020 national tariff for an ophthalmology } \\
\text { outpatient follow-up appointment with a consultant }\end{array}$ \\
\hline Ophthalmic photographer/imaging technician obtaining seven-field ETDRS images (band 6) & $£ 12.78(6.83)$ & Time cost $\times$ photographer salary+equipment cost \\
\hline Ophthalmic photographer/imaging technician obtaining UWF images (Band 6) & $£ 9.75(5.85)$ & Time cost $\times$ photographer salary+equipment cost \\
\hline Reading ETDRS images by ophthalmic grader (Band 7) & $£ 10.45(6.40)$ & Time cost $\times$ grader salary \\
\hline Reading UWF images by ophthalmic grader (Band 7) & $£ 9.10(5.77)$ & Time cost $\times$ grader salary \\
\hline Reading ETDRS images by ophthalmologist & $£ 21.58(9.02)$ & Time cost $\times$ salary \\
\hline Reading UWF images by ophthalmologist & $£ 18.23(7.28)$ & Time cost $\times$ salary \\
\hline Taking SD-OCT & Occurs in both pathways & Cost not included \\
\hline Reading SD-OCT_-grader (band 7) & $£ 4.08(3.25)$ & Time cost $\times$ salary \\
\hline
\end{tabular}

Equipment cost is a small \% of total cost (see online supplemental appendix table A2). Salary costs include employers' costs at 20\%. Reading SD-OCT by ophthalmologist is included in the NHS reference cost for the standard care pathway.

*Ophthalmologists face to face evaluation of patients with access to SD-OCT images.

ETDRS, Early Treatment Diabetic Retinopathy Study; NHS, National Health Service; SD-OCT, spectral domain optical coherence tomography; UWF, ultrawide field. 
Table 3 Relative costs of surveillance of people with previously successfully treated DMO

\begin{tabular}{|c|c|c|c|}
\hline & Sensitivity & Specificity & Cost per 100 patients \\
\hline Standard care pathway * & $\begin{array}{l}\text { Assumed } \\
100 \%\end{array}$ & $\begin{array}{l}\text { Assumed } \\
100 \%\end{array}$ & $\mathrm{f} 5800.00 \dagger$ \\
\hline Grader pathway (band 7) & $97 \%$ & $31 \%$ & $\begin{array}{l}(4.08 \times 100)+(69 \ddagger x 58)= \\
f 4410.00 \S\end{array}$ \\
\hline Cost difference & & & f1390.00ף \\
\hline
\end{tabular}

*Standard care pathway=ophthalmologists face-to-face evaluation of patients with access to SD-OCT images.

tCosts for 100 patients in the standard of care pathway.

$\ddagger$ Ophthalmologists' time of reviewing $69 \%$ of patients classified as possibly active

DMO when DMO is inactive, based on specificity.

$\S 0$ phthalmic grader costs for reading 100 OCTs+Ophthalmologist costs for $69 \mathrm{x}$

standard care visits.

ๆCurrent standard-of-care costs minus ophthalmic grader costs.

DMO, diabetic macular oedema; SD-OCT, spectral-domain optical coherence tomography.

graders could provide useful savings in ophthalmologist time. Cost savings per 100 patients could range between $£ 810.00$ (if graders were asked to identify any active DMO in either eye possibly requiring treatment) to $£ 2840.00$ (if graders were asked to identify only central involving active DMO), because of differences in specificity affecting the proportion of people referred to ophthalmologists. Specificity is the main factor driving cost differences.

\section{PDR: comparison of ophthalmic grader pathway with current standard care}

Table 4 shows costs of surveillance of PDR comparing ophthalmic grader's pathway with the standard care. Cost savings from graders reading the seven-field ETDRS and UWF images could be $£ 461.00$ and $£ 1889.00$ per 100 follow-up visits respectively, because the UWF images take less time to read. The time to obtain and read images (table 1 ) is the main driver of the differences of costs since the equipment costs were small.

Most (SENA 1 and SENA 2: online supplemental appendix, tables A8-9) corroborate the main finding that the ophthalmic grader pathway could save ophthalmologist time. Savings would be modest or even zero in SENA 4 (online supplemental table A10) for ophthalmic graders evaluating seven-field ETDRS images for people with pre-retinal or vitreous haemorrhages (ie, high-risk PDR); in this group there would be savings with UWF imaging.

PDR; comparison with enhanced reference standard

In EMERALD, both ETRDS and UWF images were examined in the enhanced standard. If the standard of care for PDR were to be supplemented with images in clinical practice, only one set of images would be used. Based on the results, we have assumed these would be UWF images.

Online supplemental appendix, table A11 shows an analysis comparing the ophthalmic graders referral for either eye by UWF imaging against standard care combined with either UWF imaging or seven-field ETDRS as graded by the ophthalmologists of active PDR in either eye. Costs using UWF imaging are lower than those using seven-field ETDRS images. So, when comparing enhanced standard care with the grader pathway, costs of UWF images are used. The grader pathway is about half the cost of the enhanced reference standard (table 5).

\section{DISCUSSION}

Follow-up of DMO after treatment by trained and tested ophthalmic graders using SD-OCT was found to have very good sensitivity (97\%), but lower specificity, ranging from $31 \%$ to $56 \%$ depending on the scenario investigated. Even that level of specificity would allow useful savings in ophthalmologist time, which could be directed for example, to the care of other people with visual threatening disease. In PDR, the overall sensitivity of the ophthalmic grader pathway was lower than that of DMO, but the sensitivity was higher in high risk PDR (pre-retinal and/ or vitreous haemorrhage), nearly reaching $90 \%$ if UWF imaging was used.

In EMERALD, images were evaluated without any information about the patient, with ophthalmic graders (and ophthalmologists undertaking the enhanced reference standard) masked to clinical data and previous images. Specificity is an important factor in the costs of the grader pathway. In routine care it is likely that having access to clinical information and previous images would improve sensitivity and specificity of the grader pathway. For example, it is possible that in the medical records the clinician would have recorded there was a macular cyst on SD-OCT at the time treatment was stopped; in that case it is likely the grader would not refer the patient. Previous images would enable for graders to check whether any lesion seen, for example, new vessels elsewhere, had been there previously, and if so, whether they were unchanged or improved/resolved, or whether they were a new finding, reducing inappropriate referrals and improving specificity. Access to medical records and previous images is now facilitated by the use of electronic medical records across the UK (eg, Medisoft, MediSIGHT, OpenEyes) and already existing and new platforms (eg, Ophthalsuite) to view fundus images and SD-OCTs.

One option would be that if the grader sees a questionable feature, annotates it (to save ophthalmologist time) and submits the image(s) (not the patient) for review by the ophthalmologist. This would avoid a proportion of potential referrals.

Table 4 Cost comparison —ophthalmic grader pathway and standard care pathway

\begin{tabular}{lllll}
\hline & Sensitivity & Specificity & Cost per 100 patients & Cost saving compared with standard care* \\
\hline Standard care pathway $+\dagger$ & Assumed 100\% & Assumed 100\% & $£ 5800.00$ & - \\
\hline Ophthalmic grader evaluating seven-field ETDRS images* & $85 \%$ & $48 \%$ & $f 5339.00$ & $£ 461.00 \ddagger$ \\
\hline Ophthalmic grader evaluating UWF images* & $83 \%$ & $54 \%$ & $£ 4611.00$ & $£ 1189.00 \S$ \\
\hline
\end{tabular}

${ }^{*}$ The analysis from which the sensitivity and specificity values are derived in this table are based on either eye being active and referrals to ophthalmologist. ${ }^{*}=$ Grader referral for PDR = 'active' + 'unsure' + 'ungradable'. The cost will not be increased if both eyes show active PDR.

tStandard care pathway=ophthalmologist face-to-face examination for active PDR in either eye.

‡Calculated as (time of taking ETDRS photos+costs of ophthalmic grader reading ETDRS photos) x100 +(52 × standard of care costs). Because of the specificity, $52 \%$ of patients will be referred to the ophthalmologist.

$\S$ Calculated as (time of taking UWF fundus images+costs of ophthalmic grader reading UWF images) ${ }^{*} 100+(46$ * standard of care costs). Because of the specificity, $46 \%$ of patients will be referred to the ophthalmologist.

ETDRS, Early Treatment for Diabetic Retinopathy Study; PDR, proliferative diabetic retinopath; UWF, ultrawide field. 
Table 5 Cost and consequence comparison of enhanced reference standard* and grader pathway

\begin{tabular}{lllll}
\hline & Sensitivity & Specificity & Cost per 100 patients & Cost savings \\
\hline Standard of care+UWF images* & Assumed 100\% & Assumed 100\% & f8598 & - \\
Ophthalmic Grader evaluating UWF imagest & $79.7 \%$ & $58.8 \%$ & $£ 4263$ & $£ 4335$ \\
\hline
\end{tabular}

* Ophthalmologists face-to-face evaluation of patients with access to SD-OCT images supplemented by the reading by the ophthalmologists of the UWF images.

tIn the main analysis, ophthalmic graders refer to ophthalmologists all patients identified as having 'active PDR', but also if they were 'uncertain' about the diagnosis of if 'ungradable' images.

UWF, ultrawide field.

A more efficient option, although more difficult to organise, would be a 'one-stop' system in which patients are imaged, images graded on the same day (an ophthalmologist could be available for advice in situ or remotely), and patient informed of the results immediately. As found in the qualitative work undertaken in EMERALD (see accompanying manuscript by Prior and Lois), this would be preferred by patients.

Given the very high number of people referred to ophthalmology clinics with DMO and PDR, clinics are finding it difficult to cope with the demand, resulting in delays in evaluation and treatment of patients. ${ }^{12}$ Delayed treatment leads not only to sight loss, ${ }^{2}$ but can reduce the cost-effectiveness of therapies. For example, a reduction in the number of anti-VEGF injections during the first year of treatment if patients are not seen and treated promptly, would lead to a lesser visual acuity improvement. ${ }^{13}$ Furthermore, as a result of these increased waiting times, the NHS often subcontracts work to private clinics or employs locum staff to cope with the demand; an expensive exercise. EMERALD showed the new pathway could save ophthalmologist time; releasing ophthalmologists to see or treat other patients could reduce the need for frequent NHS waiting list initiatives, saving money to the NHS. Whether or not, however, the ophthalmologist's time released is used efficiently in the NHS for other purposes and whether savings are realised will depend on an efficient reorganisation of services.

We used national reference costs for England of an ophthalmology outpatient appointment. These have sometimes been criticised for underestimating true costs, including at NICE Technology Appraisal Committee meetings, as noted in the NICE guidance on ranibizumab for DMO (TA 274). The true cost of an outpatient visit, however, is not known.

Times required to obtain and evaluate UWF images were shorter than those of seven-field ETDRS images with comparable sensitivity (higher for high risk PDR). This implies that if imaging is added, UWF should be used. In EMERALD, UWF images included three fields; if one were to provide comparable sensitivity and specificity, this would reduce time to obtain and grade images. In EMERALD images were obtained following pupillary dilation. If a single UWF image obtained without mydriasis provided comparable sensitivity and specificity, patient satisfaction would probably increase. Patients would be able to drive following the examination, and time in clinic would be reduced, as they would not need to wait for their pupils to dilate prior to imaging. It would also lead to some savings from dilating drops not being required.

We provided training costs for graders as observed in EMERALD. If ophthalmic grading is introduced in practice, other arrangements might be made, including distant online training. Once a comprehensive training set of images of previously treated patients had been developed, graders could learn online at their own pace. This would remove travel costs to face to face meetings, which are less appropriate in the COVID-19 era. There would be initial costs of creating sets of training images and supporting material, but there would be no costs of ophthalmologist or other trainers doing face-to-face teaching. There would be a need for a regular quality assurance system after initial training.

EMERALD did not assess the marginal benefits of adding the reading of UWF images by ophthalmologists to standard care. The marginal cost of this would be $\sim £ 28$ per patient.

If sensitivity for detecting reactivated PDR was lower than ideal and some people with active PDR were missed, the consequences would depend on the rate of progression of the disease. This would be expected to be slower in patients previously treated by PRP, as those included in EMERALD, than in those with treatment-naïve PDR. Furthermore, reactivation might be identified at the next follow-up visit before any harm resulted. Moreover, patients themselves would be likely to seek care if they were to experience floaters or sight loss, for example, due to a vitreous haemorrhage. It is possible that in the future some people with PDR will be treated with anti-VEGFs, and the rate of progression of reactivated PDR after anti-VEGF treatment is unknown. It might be faster than after PRP and the consequences more severe. ${ }^{14} 15$

In conclusion, EMERALD showed that surveillance of people with previously successfully treated DMO and PDR by ophthalmic graders using multimodal imaging can reduce costs and release ophthalmologist time.

Collaborators EMERALD Study Group: Ahmed Saad and Daniela VaideanuCollins, James Cook University Hospital, South Tees Hospitals NHS Foundation Trust; Augusto Azuara-Blanco, Centre for Public Health, Queen's University, and The Belfast Health and Social Care Trust, Belfast; Caroline Styles, Queen's Margaret Hospital, Fife; Christine McNally, Andrew Jackson and Rachael Rice, Northern Ireland Clinical Trials Unit; Clare Bailey, Bristol Eye Hospital, University Hospitals Bristol NHS Foundation Trust; Danny McAuley, Queen's University and Royal Victoria Hospital, Belfast H\&SC Trust; David H Steel, Clair Barbour and Leontia Bell, Sunderland Eye Infirmary, City Hospitals Sunderland NHS Foundation Trust; Faruque D Ghanchi and Zeid Madanat, Bradford Teaching Hospitals NHS Trust; Geeta Menon, Manju Chandran, Sely Mathews and Mohammed Galal, Frimley Park Hospital NHS Foundation Trust; Haralabos Eleftheriadis and Stefanos Efraimidis, Kings College Hospital NHS Foundation Trust; Jonathan A. Cook, Ariel Wang and William Sones, Centre for Statistics in Medicine, University of Oxford; Lindsay Prior, Centre for Public Health, Queens University, Belfast; Nachiketa Acharya, Sheffield Teaching Hospitals NHS Foundation Trust; Samia Fatum and Janette Savage, John Radcliffe Hospital, Oxford University Hospitals NHS Foundation Trust; Sobha Sivaprasad, Moorfields Eye Hospital NHS Foundation Trust; Peter H Scanlon and Katerina Ivanova, Gloucestershire Hospitals NHS Foundation Trust; Tariq M Aslam and Zaria Ali, Manchester Royal Eye Hospital, Central Manchester University Hospitals NHS Foundation Trust; Victor Chong, Royal Free Hospital NHS Foundation Trust, London.

Contributors Study concept and design: NL and NW. Data analysis: MM, HM and NW. Interpretation of data: NL, SA and NW. Drafting of the manuscript: MM, HM, NL, SA and NW which was reviewed and approved by the EMERALD study team.

Funding This work was supported by the Health Technology Assessment (HTA) of the National Institute for Health Research (NIHR), grant number 15/42/08.

Competing interests None declared.

Patient consent for publication Not required.

Provenance and peer review Not commissioned; externally peer reviewed.

Data availability statement Data are available on reasonable request. The authors would consider sharing of data result of this work upon reasonable request and following approval by the sponsor and the EMERALD Study Group. 
Supplemental material This content has been supplied by the author(s). It has not been vetted by BMJ Publishing Group Limited (BMJ) and may not have been peer-reviewed. Any opinions or recommendations discussed are solely those of the author(s) and are not endorsed by BMJ. BMJ disclaims all liability and responsibility arising from any reliance placed on the content. Where the content includes any translated material, BMJ does not warrant the accuracy and reliability of the translations (including but not limited to local regulations, clinical guidelines, terminology, drug names and drug dosages), and is not responsible for any error and/or omissions arising from translation and adaptation or otherwise.

Open access This is an open access article distributed in accordance with the Creative Commons Attribution 4.0 Unported (CC BY 4.0) license, which permits others to copy, redistribute, remix, transform and build upon this work for any purpose, provided the original work is properly cited, a link to the licence is given, and indication of whether changes were made. See: https://creativecommons.org/ licenses/by/4.0/.

\section{ORCID iD}

Noemi Lois http://orcid.org/0000-0003-2666-2937

\section{REFERENCES}

1 Mathur R, Bhaskaran K, Edwards E, et al. Population trends in the 10-year incidence and prevalence of diabetic retinopathy in the UK: a cohort study in the clinical practice research Datalink 2004-2014. BMJ Open 2017;7:e014444.

2 Foot B, MacEwen C. Surveillance of sight loss due to delay in ophthalmic treatment or review: frequency, cause and outcome. Eye 2017:31:771-5.

3 National Institute for Health and Care Excellence (NICE). Guide to the methods of technology appraisal. London: National Institute for Health and Care Excellence, 2013.

4 NICE. Aflibercept for treating diabetic macular oedema (TA346). National Institute for Health and Care Excellence, 2015.
5 Wykoff CC, Eichenbaum DA, Roth DB, et al. Ranibizumab induces regression of diabetic retinopathy in most patients at high risk of progression to proliferative diabetic retinopathy. Ophthalmol Retina 2018;2:997-1009.

6 Lois N, Cook JA, Wang A, et al. Evaluation of a new model of care for people with complications of diabetic retinopathy: the EMERALD study. Ophthalmology 2021;128:561-73.

7 Lois N, Cook J, Aldington S, et al. Effectiveness of multimodal imaging for the evaluation of retinal oedema and new vesseLs in diabetic retinopathy (EMERALD). BMJ Open 2019;9:e027795.

8 Curtis L, Burns A. Unit costs of health and social care. Canterbury: Personal Social Services Research Unit, University of Kent, 2019.

9 NHS England and NHS Improvement Joint Pricing Team. 2019/20 national tariff payment system: national prices and prices for emergency care services, 2019. Available: https://improvement.nhs.uk/resources/national-tariff/\#h2-201920-nationaltariff-payment-system [Accessed 12 Feb 2020].

10 HM Treasury. The green book: central government guidance on appraisal and evaluation, 2020. Available: https://assets.publishing.service.gov.uk/government/ uploads/system/uploads/attachment_data/file/938046/The_Green_Book_2020.pdf

11 Netten A, Curtis L. Unit costs of health and social care Canterbury: personal Socila services research unit, 2003.

12 Royal College of Ophthalmologists. Ophthalmology - the hospital eye service in crisis, 2019. Available: https://www.rcophth.ac.uk/wp-content/uploads/2019/01/RCOphthA4-Census-Infographic.pdf. [Accessed 17 Dec 2020].

13 Cantrell RA, Lum F, Chia Y, et al. Treatment patterns for diabetic macular

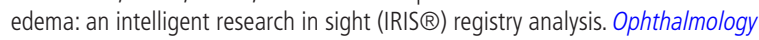
2020;127:427-9.

14 Obeid A, Su D, Patel SN, et al. Outcomes of eyes lost to follow-up with proliferative diabetic retinopathy that received Panretinal photocoagulation versus intravitreal anti-vascular endothelial growth factor. Ophthalmology 2019;126:407-13.

15 Wubben TJ, Johnson MW, Sohn EH, Anti-VEGF Treatment Interruption Study Group. Anti-Vascular endothelial growth factor therapy for diabetic retinopathy: consequences of inadvertent treatment interruptions. Am J Ophthalmol 2019;204:13-18. 